\title{
DNA Biosensors-A Review
}

\section{Kavita $\mathbf{V}$}

Department of Microbiology, Gargi College, Siri Fort Road, New Delhi, India

*Corresponding author: Kavita V, Associate Professor, Department of Microbiology, Gargi College, Siri Fort Road, New Delhi 110049, India, Tel: 9811873144; E-mail: atvikav@gmail.com

Received date: March 20, 2017; Accepted date: March 30, 2017; Published date: April 5, 2017

Copyright: (c 2017 Kavita V. This is an open-access article distributed under the terms of the Creative Commons Attribution License, which permits unrestricted use, distribution and reproduction in any medium, provided the original author and source are credited.

\begin{abstract}
Recent advances in developing biosensors which are sensitive and specific have opened new opportunities for DNA biosensors. DNA biosensors, based on nucleic acid recognition methods, are being developed towards the assay of rapid, simple and economical testing of genetic and infectious diseases. Moreover, the detection of specific DNA sequence is of significance in numerous areas including clinical, environmental and food analysis. Advancements in technology like SELEX and SAM are being used to develop better detection methods (both Direct and Indirect detection) for DNA Biosensors. Unlike enzyme or antibodies, nucleic acid recognition layers can be readily synthesized and regenerated for multiple uses. DNA biosensors and gene chips are of major interest due to their great potential for obtaining sequence-specific information in a faster, simpler and cheaper manner compared to the traditional hybridization. Further, increase of interest to DNA based sensors can be expected in near future together with a commercial production of these devices and their extensive use. However, basic research is still necessary to improve the sensor technologies, sensing strategies as well as analytical instrumentations and procedures.
\end{abstract}

Keywords: DNA; Biosensor; Nucleic acid; Hybridization; Electochemical detection complementarity.

\section{DNA Biosensors}

Biosensors have become very popular from last 20 years. New research and developments in the field of biosensor play important roles in daily life. In recent years, biosensors have been increasingly used for continuous monitoring of biological and synthetic processes used in industrial and clinical chemistry. Biosensor is becoming popular in the field of food analysis [1], bioterrorism [2,3], environmental [2-4] and in the area of human health monitoring and diagnostics [5-7].

In general, biosensor is small device employing biological recognition properties for a selective bio-analysis [8-10]. Such devices rely on the intimate coupling of a biological recognition element with a physical transducer to convert the biological signals into an electrical signal or other signals, proportional to the concentration of analytes [11-16]. Biosensors eliminate the need of the sample preparation and hence offer great promise for several on-site analytical applications of rapid and low cost measurements [17].

A basic biosensor assembly includes a receptor, transducer and processor. The sensing elements may be whole cells, antibodies, enzymes or nucleic acids forming a recognition layer that is integrated with transducer via immobilization by adsorption, cross-linking or covalent binding. The transducers are based upon the parameters of measurement. It may be amperometric (current measurement at constant potential) [18], potentiometric (potential measurement at constant current) [19], piezoelectric (measurement of changes in mass) [20], thermal (measurement of changes in temperature) [21] or optical (detect changes in transmission of light) [22]. The usual analytical techniques require a number of steps, much labor, time and expensive instruments whereas biosensors are quick, simple, economical and may be used in small hospitals and laboratories of remote areas where sophisticated instrument facilities are not available.

In recent years, nucleic acids have been extensively used into a wide range of biosensors and bio analytical assays, due to their wide range of physical, chemical and biological activities $[23,24]$. In nucleic acid based biosensors, sensing elements are oligonucleotides, with a known sequence of bases, or a fragment of DNA or RNA. Nucleic acid biosensors are either based on the highly specific hybridization of complementary strands of DNA/RNA molecules or play the role of a highly specific receptor of biochemical/chemical species [25-28]. Nucleic acid biosensors are of major interest owing to their great promise for obtaining the sequence-specific information in a faster, simpler and cheaper manner compared to the traditional ones. Unlike enzymes or antibodies, nucleic acid recognition layers can be readily synthesized and regenerated for multiple uses. Nucleic acid biosensors can be more sensitive and specific when combined with polymerase chain reaction (PCR) methods (Bell and Ranford-Cartwright) [29,30].

\section{DNA Hybridisation Biosensors}

Complementary DNA base pairing is the basis for the biorecognition process in hybridization biosensors. Short, $20-40$ base pair highly target-selective single-stranded DNA segments are immobilized on the electrode surface. The DNA fragments have to be immobilized in a way that retains their stability, reactivity, accessibility to target analyte and optimal orientation. An electrical signal is produced when target DNA binds to the complementary sequence of the capture or probe DNA in a process called hybridization. Ferrocenyl naphthalene diimide (FND) is an example of an electrochemical indicator that binds preferentially to the DNA duplexes and this results in an electrochemical signal. Horseradish Peroxidase and alkaline phosphatase are some other enzyme labels which are used as a measure of hybridization. Colloidal gold has also been used as a hybridization label. Like other complex biological macromolecules, the experimental 
Page 2 of 5

conditions, such as temperature, ionic strength, and time allowed for hybridization, have to be controlled in order to achieve high selectivity and sensitivity.

Single-stranded, 15-40 bases long DNA or RNA oligonucleotide sequences (aptamers) are rapidly screened in the SELEX (systematic evolution of ligands by exponential enrichment) process for their ability to selectively bind low molecular weight organic, inorganic or protein targets. In solution, the synthetic nucleotide chains form intra molecular interactions that fold the aptamers molecules into a complex three-dimensional shape. The unique shape of the aptamer allows it to bind tightly and selectively with its target molecule. Aptamers can either bind to small sections of macromolecules, such as proteins, or they can engulf a small molecular target.

Chosen aptamers after several cycles of SELEX can also be chemically modified to increase their stability and affinity for a target molecule. Aptamers are sensitive to their environment and have to be protected from high temperatures and DNAase enzymes.

One design of a commercialized DNA biosensor is based on the selective reaction between a DNA capture probe immobilized on the electrode surface and target DNA in the sample.

The biosensor uses a sandwich type assay as shown in capture probe (or probes) and an insulator component. The DNA capture probe is immobilized on the gold using an alkane thiol linker that projects it beyond a layer of shorter alkane thiols, which covers the self-assembled monolayer (SAM) technology which is used to create the chemical layer attached to the gold electrode (Figure 1). The monolayers are mixed SAMs, each comprised of a sequence specific surface between the DNA capture probes and thereby minimizes interference from redox active materials in the sample and nonspecific adsorption, by blocking their access.

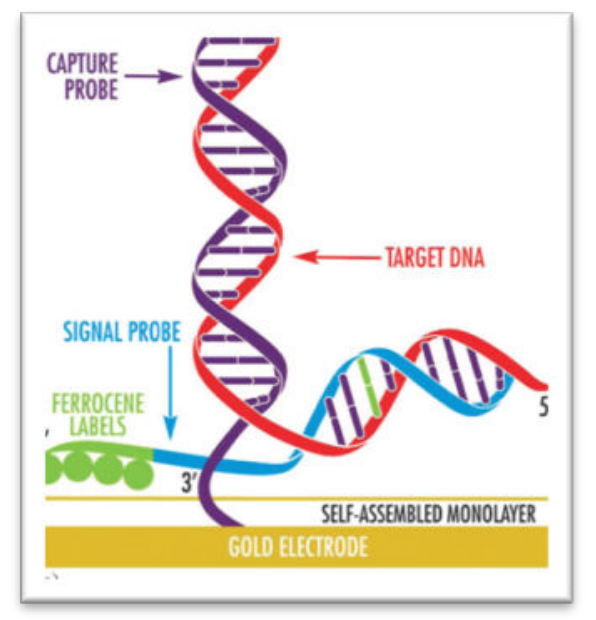

Figure 1: Self assembled monolayer.

Exposing electrode to the sample results in hybridization between the capture probe and complementary strand of the target DNA (Figure 2).

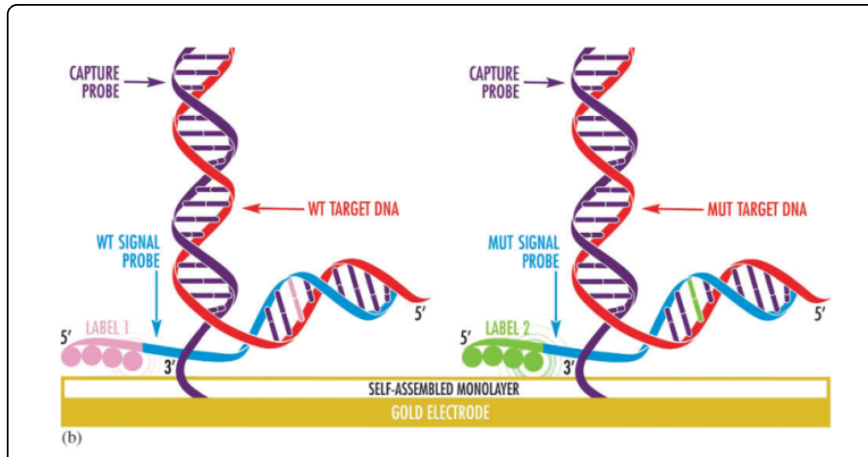

Figure 2: Strand of the target DNA.

\section{SPR-DNA Biosensor}

Aptamers are short fragment of single-strand DNAs (ssDNAs)/ RNAs selected in vitro and can bind with a broad range of target molecules like amino acids, drugs, proteins and other molecules with high affinity and specificity. These aptamers are screened from extremely complex libraries of nucleic acids through a process called Systematic Evolution of Ligands by Exponential enrichment (SELEX). Nakamura et al. developed a SPR bio-sensor based on screened DNA aptamers having capability to bind micro-cystin very specifically. Screening was done by the in vitro selection method of twelve rounds and obtained a sorbent specifically suitable for micro-cystin detection. The sensitivity and precision of micro-cystin detection was not as high compared with the methods reported previously and can be further improved by using high affinity aptamers.

\section{Electrochemical DNA Biosensors}

The detection techniques play very important role in design of biosensors and are selected according to their specific application. Among the various devices designed so far, electrochemical DNA Biosensors have attracted more attention due to their high sensitivity and rapid response. Electrochemical devices are very useful for sequence-specific bio-sensing of DNA. The miniaturization of devices and advanced technology make them excellent tool for DNA diagnostics. Electrochemical detection of DNA hybridization usually involves monitoring a current at fixed potential. Electrical modes were developed for detection of both label-free and labeled objects [31-42]. The immobilization of the nucleic acid probe onto the transducer surface plays an important role in the overall performance of DNA biosensors and gene chips [43-45].

The immobilization step requires a well-defined probe orientation and accessible to the target for hybridization. Depending upon the nature of the physical transducer, various methods can be used for attaching the DNA probe to the solid surface such as the use of thiolated DNA probe for self-assembled monolayers (SEM) onto gold transducers by covalent linkage to the gold surface via functional alkanethiol-based monolayers. The other method of attachment of DNA probe is to biotinylate DNA probe and attachment through biotin-avidin interaction on electrode surface [30-32,46]. The avidin modified polyaniline electrochemically deposited onto a Pt disc electrode for direct detection of $E$. coli by immobilizing a 5' biotin labeled probe using a differential pulse volta metric technique in the presence of methylene blue as a DNA hybridization indicator [14,31]. 
Page 3 of 5

Similarly, electrochemical DNA biosensor based on poly pyrrolepolyvinyl sulfonate coated onto Pt disc electrode was also fabricated using biotin-avidin binding [32].

The discovery of carbon nanotubes (CNTs) in DNA analysis plays an important role by development of electrochemical DNA biosensor. CNT not only enables immobilization of DNA molecules but also used as powerful amplifier to amplify signal transduction of hybridization. CNT also works as novel indicator of hybridization. The application of arrayed CNT into DNA chip require small amount of sample and development of CNT based biosensor play major role on DNA based diagnostics in hospitals or at home [47].

The knowledge of peptide nucleic acid (PNA) has opened a new research area of DNA biosensors. PNA is a DNA mimic in which the sugar phosphate backbone is replaced with a pseudopeptide. The unique structural, hybridization and recognition features of solutionphase PNA can be readily extrapolated onto transducer surfaces in connection with the design of highly-selective DNA biosensors. Such use of surface-confined PNA recognition layers imparts remarkable sequence specificity onto DNA biosensors including detection of single-base mismatches [45].

The hybridization is commonly detected by the increase in current signal due to redox indicator (that recognizes the DNA duplex) or from other hybridization-induced changes in electro chemical parameters (e.g. conductivity or capacitance). New redox indicators, offering greater discrimination between single strand (ss) and dsDNA $[27,29,33,34,36,37,48]$. The use of an inter calator ferro cenyl naphthalene di-imide that binds to the DNA hybrid more tightly than usual inter calators and displays small affinity to the single-stranded probe [49]. The electrochemical DNA biosensor may be labeled based and lebeled free.

\section{Label Based or Indirect Detection}

In label based electrochemical biosensor specific organic dyes, metal complexes or enzymes are used for hybridization detection. The use of enzyme labeled probe offers great promise for electro chemical detection of DNA hybridization [50]. On addition of substrate to the enzyme modified electrode surface, the electrochemical activity of the product simplifies the detection of hybridization [51-53].

Redox- active molecules such as dauno mycin, methylene blue which is inserted between the dsDNA and gives signal which can be used for hybridization detection [39,50]. Redox-active molecules based two commercialized DNA chips have been introduced in molecular diagnosis market in the trade name of eSensor TM produced by Motorola Life sciences [25], Inc. and Genlyser TM by Toshiba [26].

\section{Label Free or Direct Detection}

Contrary to indirect detection techniques, where labeling is a requirement to translate the hybridization event into a signal, in direct detection techniques, a target molecule or any other object from the system does not need to be labelled $[39,40]$. Although label-dependent methods achieve the highest sensitivities, eliminating the labeling steps simplifies the readout, the speed and ease of nucleic acid assays.

In a label-free method the immobilized probe recognizes a complementary sequence if the target is present in the sample. Next, the transducer converts the biological interaction into a measurable signal, proportional to the degree of hybridization that is to the amount of target molecule in the sample. Label-free strategies reduce analysis times and cost. They are also free from un-favorable effects from the labels, such as its instability and steric hindrances $[54,55]$.

Recently, a new label-free electrochemical detection technique has been developed which is faster and simpler [14,15,30,32,38]. It is possible to exploit changes in the intrinsic electroactivity of DNA (guanine oxidation peak of hybridization). To overcome the limitations of the probe sequences(absence of $G$ ), guanines in the probe sequence were substituted by inosine residues (pairing with $\mathrm{C}$ ) and the hybridization was detected through the target DNA guanine signal $[15,38]$. Changes in the guanine oxidation, and of other intrinsic DNA redox signals, have thus been used for detecting chemical and physical damage. A greatly amplified guanine signal, and hence hybridization response, was obtained by using the $\mathrm{Ru}$ (bpy) 3 redox mediator. Direct, label-free, electrical detection of DNA hybridization has also been accomplished by monitoring changes in the conductivity of conducting polymer molecular interfaces (DNA-modified poly pyrrole films). Eventually, it would be possible to eliminate these polymeric interfaces and to exploit different rates of electron-transfer through ss DNA and ds DNA for probing hybridization (including mutation detection via the perturbation in charge transfer through DNA). Although elctrochemical DNA biosensors based on the use of redox indicator provided reliable and precise information in mutation detections redox probes require high potential that are prone to interferences and often destroy hybrid double strand structure. To overcome this problem, an electrochemical DNA biosensor based upon the concept of metal enhanced detection for the determination of Micro cystis spp. was developed. The biosensor was constructed by immobilizing 17-mer DNA on a gold electrode via avidin- biotin chemistry.

\section{Applications of DNA Biosensors}

The detection of specific DNA sequence is of significance in many areas including clinical, environmental and food analysis $[7,23,24]$. The analysis of gene sequences and the study of gene polymorphisms play a fundamental role in rapid detection of genetic mutations, offering the possibility of performing reliable diagnosis even before any symptoms of a disease appear. In environmental and food areas the detection of specific DNA sequences can be used for the detection of genetically modified organism (GMO) or pathogenic bacteria.

DNA biosensors and gene chips are of major interest due to their tremendous promise for obtaining sequence-specific information in a faster, simpler and cheaper manner compared to the traditional hybridization $[25,26]$. Recent advances in developing such devices opens new opportunities for DNA diagnostics. DNA biosensors, based on nucleic acid recognition processes, are rapidly being developed towards the assay of rapid, simple and economical testing of genetic and infectious diseases. Unlike enzyme or antibodies, nucleic acid recognition layers can be readily synthesized and regenerated for multiple use. DNA sensors can be made by immobilizing single stranded (ss) DNA probes on different electrodes using electro active indicators to measure the hybridization between DNA probes and their complementary DNA strands [27-29].

The current method for the identification of specific DNA sequence in biological samples are based on isolation of double stranded (ds) genomic DNA and further polymerase chain reaction (PCR) to amplify the target sequence of DNA. The PCR products can be subjected to electrophoresis or may beads or bed onto a suitable membrane and exposed to a solution containing DNA probe (Southern Blot). The DNA probe is either chemically or enzymatically 
labeled with radioactive material, chemilumnophore or ligands such as biotin etc. as the nucleic acid itself has not able to provide any signal. Recent advances in the field of bio molecular techniques can be used to fabricate new generation miniaturized biosensor.

\section{Conclusions and Future Prospects}

From the first discovery of electrochemistry of nucleic acids by Palecek at the end of the 1950's [51], huge progress can be observed, particularly at the development of electrochemical DNA biosensors based on the nucleic acid as bio-recognition element. Different types of electrodes immobilized with specific probes can be used to detect the presence of complementary target sequence by hybridization technique. Besides the different immobilization methods, electro active hybridization indicators (metal complexes, dauno mycin, methylene blue, etc.) and different conducting polymer based nano composites are also used for development of electrochemical biosensors.

SPR, Quantum-Dot and piezoelectric biosensors are the emerging area of molecular diagnosis. The Intelligent opto sensors interfacing based on universal frequency-to-digital converter has opened new opportunities for development of DNA biosensors [52]. Some success has been achieved in the commercialization of optical fiber sensors. However, they still suffer from competition with other mature sensor technologies and new ideas are being continuously developed and tested not only for the traditional measurements but also for new applications [53,54].

The use of DNA bio strip and biochip technologies eliminates the role of PCR. Future biosensors will require the development of new reliable devices or the improvement of the existing ones in order to allow superior transduction, amplification, processing, and conversion of the biological signals. Nanotechnology is playing an important role in development of efficient biosensors for the toxin detection. Different types of nano materials (nanoparticles and nanotubes) with different properties have been used. They offer exciting opportunities to improve the performance of electo-chemical biosensors for the detection of micro cystins.

Efficient biosensors will not necessarily function as a stand-alone detector, but will form an integral part of an analytical system. Compact and portable devices will constitute another future area of intensive multidisciplinary sensor research. Further, increase of interest to DNA based sensors can be expected in near future together with a commercial production of these devices and their wide use.

However, basic research is still necessary to improve the sensor technologies, sensing strategies as well as analytical instrumentations and procedures.

\section{References}

1. Eden-Firstenberg R, Schaertel BJ (1988) Biosensor in the food industry: Present and future. J of Food Protection 51: 811-820.

2. Lindner D (2001) Lab as a chip.

3. Burkle FM (2003) Measures of effectiveness in large scale bioterrorism events. Prehosp Disast Med 18: 258-262.

4. Maseini M (2001) Affinity electrochemical biosensors for pollution control. Pure Appl Chem 73: 23-30.

5. Malhotra BD, Chaube A (2003) Biosensors for clinical diagnostics industry. Sensor Actuators B 9: 117-126.

6. Annelies B, Dirk B, Michel L (2005) Clinical and analytical performance of the Accu-chek inform point-of-care glucose water. Poin of Care 4: $36-40$.
7. Anjum V, Pundir CS (2007) Biosensors: Future analytical tools. Sensors \& Transducers Journal 76: 937-944.

8. Blonder R, Levi S, Tao G, Ben-Dov I, Willner J (1997) Development of amperometric and microgravimetricimmunosensors and reversible immunosensors using antigen and photoisomerizable antigen monolayerelectrodes. J Am Chem Soc 119: 467- 478.

9. Blonder R, BenDov I, Dagana A, Willner I, Zisman E (1997) Photochemically activated electrodes: Application in design of reversible immunosensors and antibody patterned interfaces. Biosens Bioelectron 12: 627-644.

10. Prummond TG (2003) Electrochemical DNA sensors. Nature Biotechnol 21: 1192-1199.

11. Wang J, Rivas G, Cai X, Florenda V, Peter EN, et al. (1997) DNA electrochemical biosensors for environmental monitoring: A review. Analytica Chimica Acta 347: 1-8.

12. Bagni G, Osella D, Sturchio E, Mascini M (2006) Deoxyribonucleic acid (DNA) biosensors for environmental risk assessment and drug studies. Anal ChimActa 574: 81-89.

13. Chikashi N, Teruaki K, Masato M, Makoto S, Jun Miyakea (2001) Usage of a dna aptamer as a ligand targeting microcystin. Molecular Crystals and Liquid Crystals 371: 369-374.

14. Arora K, Prabhakar N, Chand S, Malhotra BD (2007) Ultrasensitive DNA hybridization biosensor based on polyaniline. Biosens Bioelectronics 23: 613-620.

15. Prabhakar N, Arora K, Singh SP, Pandey MK, Singh H, et al. (2007) Polypyr role-polyvinylsulphonate film based disposable nucleic acid biosensor. Anal Chim Acta 589: 6-13.

16. Berdat D, Marin A, Herrera F, Gijs MAM (2006) DNA biosensors using fluorescence microscopy andimpedance spectroscopy. Sensors Actuators 118: 53-59.

17. Sharma K, Sehgal N, Kumar A (2003) Biomolecules for development of biosensors and their application. Curr Appl Phys 3: 307-316.

18. Ho KC, Cheu CY, Hsu HC, Cheu LC, Shiesh SC, et al. (2004) Amperometric detection of morphineat a prussian blue modified indium tin oxide electrode. Biosens Bioelectron 20: 3-8.

19. Wang J, Xu DK, Kawde AN, Polsky R (2001) Metal nanoparticle-based electrochemical stripping potentiometric detection of DNA hybridization. Anal Chem 73: 5576-5581.

20. Bunde RL, Jarvi EJ, Rosentreter JJ (1998) Piezoelectric quartz crystal biosensors. Talanta 46: 1223-1236.

21. Mebravar M, Chris B, Scharer JM, Luong JTH (2000) Fiber optic biosensors: Trends and advances. Anal Sci 16: 677-692.

22. Ivniski D, Hamid IA, Atanasov P, Wilkins E (1999) Biosensors for detection of pathogenic bacteria. Biosens Bio-electron 14: 559-624.

23. Berney H, West J, Haefele H, Alderman J, Lane W, et al. (2000) DNA diagnostic biosensor development, characterization and performance. Sens Actuators B 68: 100-108.

24. www.motorola.com/Lifesciences

25. www.dna-chip.toshiba.co.jp/eng/

26. Erdem A, Kesman K, Mesie B, Akarea US, Osoz M (2000) Novel hybridization indicator methylene blue forthe electrochemical detection of short DNA sequence related to Hepatitis B Virus. Anal Chim Acta 423: 139-149.

27. Campbell CN, Gal D, Cristler N, Banditrat C, Heller A (2002) Enzyme amplified amperometric sandwich test for RNA and DNA. Anal Chem 74: $158-162$.

28. Millan KM, Mikkelsen SR (1993) Sequence selection biosensor for DNA based on electro active hybridization indicators. Anal Chem 65: 2317-2324.

29. Prabhakar N, Arora K, Singh SP, Singh H, Malhotra BD (2007) DNA entrapped polypyrrole-polyvinyl sulfonate film for application to electrochemical biosensor. Anal Biochem 366: 71-79.

30. Arora K, Prabhakar N, Chand S, Malhotra BD (2007) Escherichia coli genosensor based on polyaniline. Anal Chem 79: 6152-6158. 
31. Arora K, Prabhakar N, Chand S, Malhotra BS (2007) Immobilization of single stranded DNA probe ontopolypyrrole-polyvinyl sulfonate for application to DNA hybridization biosensor. Sensors Actuators B 126: 655-663.

32. Yau HCM, Chan HL, Yang M (2003) Electrochemical properties of DNAintercalating doxorubicin andmethylene blue on n-hexadecylmercaptandoped 5'-thiol-labeled DNA-modified gold electrodes. Biosensors Bioelectronics 18: 873-879.

33. Gu J, Lu X, Ju H (2002) DNA sensor for recognition of native yeast DNA sequence with methylene blue as an electrochemical hybridization indicator. Electro Analysis 14: 949-953.

34. Reddy RRK, Chadha A, Bhattacharya E (2001) Porous silicon based potentiometric triglyceride biosensor. Biosens Bioelectron 16: 313-317.

35. Kara P, Kerman K, Ozkan D, Meric B, Erdem A, et al. (2002) Electrochemical genosensor for the detection of interaction between methylene blue and DNA. Electrochem Commun 4: 705-709.

36. Erdem A, Kerman K, Meric B, Ozsoz M (2001) Methylene blue as a novel electrochemical hybridization indicator. Electro analysis 13: 219-223.

37. Arora K, Malhotra BD (2008) Applications of conducting polymer based DNA biosensors. Appl Phys in the21st century, pp: 1-20.

38. Kerman K, Kobayashi M, Tamiya E (2004) Recent trends in electrochemical DNA biosensor technology. Meas Sci Technol 15: 1-11.

39. Lucarelli F, Tombelli S, Minunni M, Marrazza G, Mascini M (2008) Electrochemical and peizoelectric DNA bio-sensor for hybridization detection. Anal Chim Acta 609: 139-159.

40. Cheng G, Zhao J, Tu Y, He P, Fang Y (2005) A sensitive DNA electrochemical biosensor based on magnetite with a glassy carbon electrode modified by multi-walled carbon nanotubes in poly-pyrrole. Anal Chim Acta 533: 11-16.

41. Mascini M, Palchetti I, Murazza G (2001) DNA electrochemical biosensor. Fresenius J Anal Chem 369: 15-22.

42. Chrisey LA, Lee GU, Ferrall CEO (1996) Covalent attachment of synthetic DNA to self-assembled monolayer films. Nucl Acids Res 24: 3031-3039.
43. Marks RS, Lowe CR, Cullen DC, Weetal HH, Karube I, et al. (2007) Hanbook of Biosens Biochips. John Willey \& Sons, Chechister, UK.

44. Wang J (2008) From DNA biosensors to gene chips. Nucl Acid Res 28 3011-3016.

45. Marrazza G, Chinella I, Mascini M (1999) Disposable DNA electrochemical sensor for hybridization detection. Biosens Bio-electron 14: 43-51

46. Pingang H, Ying X, Yuzhi F (2006) Application of carbon nanotubes in electrochemical DNA biosensor(Review). Microchim Acta 152: 175-186.

47. Yang W, Ozsoz M, Hibbert DB, Gooding JJ (2002) Evidence for the direct interaction between methylene blue and guanine bases using DNAmodified carbon paste electrodes, Electro analysis 14: 1299-1302.

48. Yanmashita K, Takagi M, Kondo H, Takenaka S (2002) Electrochemical detection of nucleic base mismatches with ferrocenyl naphthalene diimide. Anal Biochem 306: 188-196.

49. Tombelli S, Mascini M, Braccini L, Anichini M, Turner APF (2000) Coupling of a DNA piezoelectricbiosensor and polymerase chain reaction to detect apolipoprotein- E polymorphisms. Biosens Bioelectron15: 363-370.

50. Palecek E (1960) Oscillographic polarography of highly polymerized deoxyribonucleic acid. Nature 188: 656-657.

51. Yurish SY (2005) Intelligent opto sensors interfacing based on universal frequency-to-digital converter. Sens Transducers Journal 56: 326-334.

52. Nazario B (2001) An introduction to fiber-optic sensors. Sensors Magazine.

53. Byoungho L (2003) Review of the present status of optical fiber sensors. Optical Fiber Technology 9: 57-79.

54. Owino IO, Mwilu SK, Sadik OA (2007) Metal enhanced biosensor for genetic mismatch detection. Analytical Bio-chemistry 369: 8-17.

55. Bell AS, Ranford-Cartwright LC (2002) Real-time quantitative PCR in parasitology. Trends Parasitol 18: 337-342. 